\title{
Comparison of Intermittent and Continuous Photother- apy to Treat Non-hemolytic Moderate Hyperbilirubine- mia in Term Infants: A Randomized, Controlled Trial
}

\author{
Roya Taheritafti ${ }^{*}$ and Moj Taheritafti ${ }^{2}$ \\ ${ }^{1}$ Department of Neonatology, Tehran, Iran \\ ${ }^{2}$ Department of Cardiology, Shiraz, Iran \\ *Corresponding author: Roya Taheritafti, Department of Neonatology, Loghman Hospital Kamali Street, Tehran, Iran
}

\section{ARTICLE INFO}

Received: 业 December 16, 2019

Published: 幽January 06, 2020

Citation: Roya Taheritafti, Moj Taheritafti. Comparison of Intermittent and Continuous Phototherapy to Treat Non-hemolytic Moderate Hyperbilirubinemia in Term Infants: A Randomized, Controlled Trial. Biomed J Sci \& Tech Res 24(2)-2020. BJSTR. MS.ID.004017.

Keywords: Jaundice; Neonate; Intermittent; Continuous; Phototherapy; Melatonin
ABSTRACT

Background: The current randomized controlled trial (RCT) aimed at investigating the efficacy of intermittent and continuous phototherapy to reduce bilirubin in neonatal jaundice and neonatal circadian rhythm.

Methods: The current double blinded RCT was conducted on 60 icteric term neonates from November 2016 to June 2017. A total of 60 icteric term neonates were randomly divided into two groups. In the continuous group, the phototherapy device was turned on for 24 hours and in the intermittent phototherapy group, the phototherapy device was turned on for 18 hours (off for eight hours). Inclusion criteria were term neonates with non-hemolytic hyperbilirubinemia admitted to the neonatal ward of Loghman Hospital. Both early and late complications were compared including the length of hospital stay, duration of phototherapy, the rate of kernicterus, decreasing rate of bilirubin after 24 and 36 hours and serum melatonin.

Results: The length of hospital stay was $2.3 \pm 0.60$ and $2.46 \pm 0.93$ days in the continuous and intermittent groups, respectively $(\mathrm{P}=0.516)$. The duration of phototherapy was $45.26 \pm 16.39$ and $46 \pm 11.82$ hours in the continuous and intermittent groups, respectively, and they had no significant differences $(\mathrm{P}=0.843)$. The rate of serum bilirubin cessation in the two groups was similar after 36 hours. Although the melatonin level was higher in the intermittent group than in the continuous group, the difference was not statistically significant $(\mathrm{P}=0.455)$.

Conclusion: According to the current study results, intermittent phototherapy was as effective as continuous phototherapy to treat icteric full-term neonates. The melatonin level in the two groups had no significant differences. However, more studies are needed to evaluate the phototherapy effects on the circadian rhythm of neonates.

\section{Short Communication}

One of the most useful and effective forms of therapy for neonatal icterus is phototherapy. Phototherapy is an available method that reduces the total serum bilirubin concentration in nearly all icteric neonates. Phototherapy, with three independent mechanisms, can reduce the total serum bilirubin concentration in neonates (photoisomerization, structural isomerization, and photooxidation). The photoisomerization pathway is responsible for more than $80 \%$ of bilirubin elimination during phototherapy [1].
The photo isomers constitute about $20 \%$ of the total bilirubin 8-12 hours after phototherapy [2]. Following the phototherapy, photo isomers of bilirubin are produced in the skin layers. On the other hand, the re-generation of bilirubin in the skin takes about one to three hours [2]. Standard tests do not detect naturally occurring bilirubin and photo isomers; thus, bilirubin levels may not reduce although the phototherapy makes bilirubin less toxic [2]. There is no definite evidence that continuous phototherapy decreases 
serum bilirubin levels more rapidly than intermittent phototherapy treatment [3]. When bilirubin levels are not approaching the exchange transfusion levels, phototherapy can be discontinued for up to one hour at frequent intervals to allow mothers feed and clean their baby without affecting the amount of bilirubin [4].

The intermittent phototherapy is useful for hospitals that their equipment is not proportional to the number of their patients requiring phototherapy. The intermittent phototherapy also helps to maintain mother-infant bonding; it is useful to maintain breastfeeding. Many clinical trials showed that the intermittent phototherapy can reduce the newborn's bilirubin as much as a continuous phototherapy did; although other studies reported contradictory results. The intermittent phototherapy may be safer due to maintaining the circadian rhythm. The continuous phototherapy may suppress the circadian rhythm. The circadian rhythmicity is affected by the sensory environment. In utero, the circadian rhythm of the fetus is well-stabilized at the end of the second trimester. The neural mechanisms to respond to a circadian light stimulus are developed early in the third trimester [5]. After birth, the diurnal rhythm of infants is not well established due to various reasons, especially in infants admitted to the neonatal intensive care unit (NICU) with continuous lighting or in the ones under phototherapy lamps. Melatonin or N-acetyl-5methoxytryptamine is produced by the pineal gland in humans. It regulates the circadian rhythm or sleep and wakefulness cycles.

The melatonin production is affected by the daily onset of darkness [6]. It is synthesized in the pineal gland during the dark cycle, whereby it conveys its chronobiologic properties. Melatonin secretion is regulated by the production of norepinephrine. Melatonin is immediately destroyed on exposure to light [7]. (Table 1) The production of melatonin is resumed in the evening at the point called the dim-light melatonin onset [8]. As mentioned by Hickman $\mathrm{AB}$ et al. [9] blue light, especially in 460 to $480 \mathrm{~nm}$ wavelengths, suppresses melatonin. Therefore, the continuous phototherapy may suppress the melatonin production since the spectrum of blue light, which is most effective (especially around $460-490 \mathrm{~nm}$ ) in reducing the bilirubin in icteric neonates, is used for phototherapy. As mentioned by Poeggeler et al., besides its function as a synchronizer of the biological clock, melatonin is a good scavenger of free radicals and is an antioxidant as well [10]. Melatonin is an antioxidant that at high levels stimulates and at low levels reduces the number of immune system parameters [11]. (Figure 1) Melatonin can easily cross the blood-brain barrier of humans [10]. Therefore, authors believe that the regulation of exposure to light at day and night in neonates is important, especially for the neonates under phototherapy. On this basis, in the current study, the intermittent phototherapy group (group 2) of icteric neonates received phototherapy for 18 hours on and eight hours off (from I 0:00 P.M to 6:00 A.M) in order to save the circadian rhythm. This group was compared with a continuous group (group 1) that received phototherapy for 24 hours.

\section{Hospital length of stay (Day)}

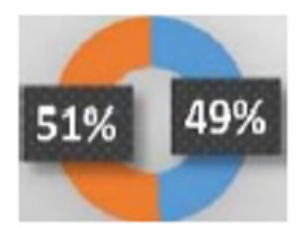

- Inter

Figure 1: Hospital length of stay in two groups.

Table 1: Topographic Index in two groups. Co: Continuous, Inter: Intermittent.

\begin{tabular}{|c|c|c|c|c|c|}
\hline Groups & \multicolumn{2}{|c|}{$\mathbf{N}$} & Mean & Std. Deviation & Std. Error Mean \\
\hline \multirow{2}{*}{ Gestationai. Age(w) } & Co & 30 & 38.57 & 0.898 & 0.164 \\
\hline & Inter & 30 & 38.67 & 1.124 & 0.205 \\
\hline \multirow{2}{*}{ Neonatai. Age(Day) } & Co & 30 & 3.47 & 1.795 & 0.328 \\
\hline & Inter & 30 & 4.83 & 1.821 & 0.332 \\
\hline \multirow{2}{*}{ Birth Weight (gr) } & Co & 30 & 3322.33 & 527.745 & 96.353 \\
\hline & Inter & 30 & 3256.33 & 382.915 & 69.91 \\
\hline \multirow{2}{*}{ Neonatal Height (em) } & Co & 30 & 49.77 & 1.331 & 0.243 \\
\hline & Inter & 30 & 49.8 & 1.448 & 0.264 \\
\hline \multirow{2}{*}{ Neonatal Head Size (em) } & Co & 30 & 34.617 & 1.3938 & 0.2545 \\
\hline & Inter & 30 & 34.567 & 0.888 & 0.1621 \\
\hline \multirow{2}{*}{ Total Bilirubin before photo (mg/dl) } & Co & 30 & 15.97 & 1.55655 & 0.28419 \\
\hline & Inter & 30 & 16.14 & 1.57996 & 0.28846 \\
\hline \multirow{2}{*}{ Direct Bilirubin before photo $(\mathrm{mg} / \mathrm{dl})$} & Co & 30 & 0.413 & 0.0681 & 0.0124 \\
\hline & Inter & 30 & 0.663 & 0.9489 & 0.1732 \\
\hline
\end{tabular}




\section{Methods and Subjects}

The current study was a prospective, randomized, controlled trial. Sixty icteric term neonates with indirect hyperbilirubinemia admitted to the NICU of Loghman Medical Center in Tehran, Iran were enrolled in the current study and informed consent was obtained from their guardians. The current study was approved by the Ethics Committee of Shahid Beheshti University of Medical Sciences in 2016 (code: 66001089/27). The study was registered in the Iranian Registry of Clinical Trials (code number: IRCT2015112225184Nl). Inclusion criteria were term neonates (gestational age $\geq 37$ weeks) with non-hemolytic hyperbilirubinemia admitted to the neonatal department of Loghman Hospital from November 2016 to June 2017. Infants were excluded for any of the following reasons: hemolytic anemia, prematurity, sepsis, severe hyperbilirubinemia (total bilirubin $>18 \mathrm{mg} / \mathrm{dL}$ ), direct hyperbilirubinemia, and the onset of jaundice within the first 24 hours of life. Chi-square test or the Fisher exact test was used to analyze the categorical data. T test and repeated measures analysis of variance (ANOVA) were used to analyze the quantitative data (Figure 2).

\section{GENDER DISTRIBUTIO

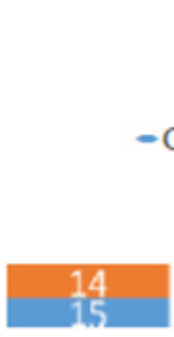 \\ FEMALE

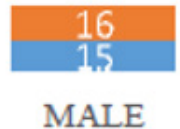

Figure 2: Gender distribution in two groups.

From November 2016 to June 2017, sixty icteric neonates via block randomization were divided into two groups. The continuous phototherapy group (group 1) received phototherapy for 24 hours. The intermittent phototherapy group (group 2) received phototherapy for 18 hours on and eight hours off (from 10:00 P.M to 6:00 A.M). Phototherapy lights were turned off during breastfeeding, changing, and changing of diapers for up to one hour in the two groups with intervals, as needed, because this procedure does not significantly reduce the effect of phototherapy [1]. (Table 2) The phototherapy device was the same in the two groups and serum bilirubin of the patients was tested every six hours. The phototherapy units with eight special blue to green tubes were employed (XHZ-90, Ningbo David Medical Devices). They were placed $30-40 \mathrm{~cm}$ above the infants in the hospital bassinet to illuminate the maximal body surface area. This device produced an average irradiance of $30 \mathrm{flW} / \mathrm{cm} 2$ with the wavelength of 425 to $475 \mathrm{~nm}$ to reduce serum bilirubin of neonates. Environmental lighting was controlled during the day and night in the intermittent group (the lamps were turned off at night at 10:00 P.M and turned on at 6:00 A.M). (Figure 3) It was tried to control light at night and day as much as possible. The melatonin was checked with enzymelinked immunosorbent assay (ELISA) (IBL, International GmbH Flughafenstr 52A and 22335; Hamburg Germany) at 8:00 A.M after the reduction of serum total bilirubin and comparison of the amounts of serum melatonin (with a normal value of 3.8-8.4 pg/mL in healthy subjects) in the two groups. In both arms, phototherapy continued until total serum bilirubin reached $<11 \mathrm{mg} / \mathrm{dL}$.

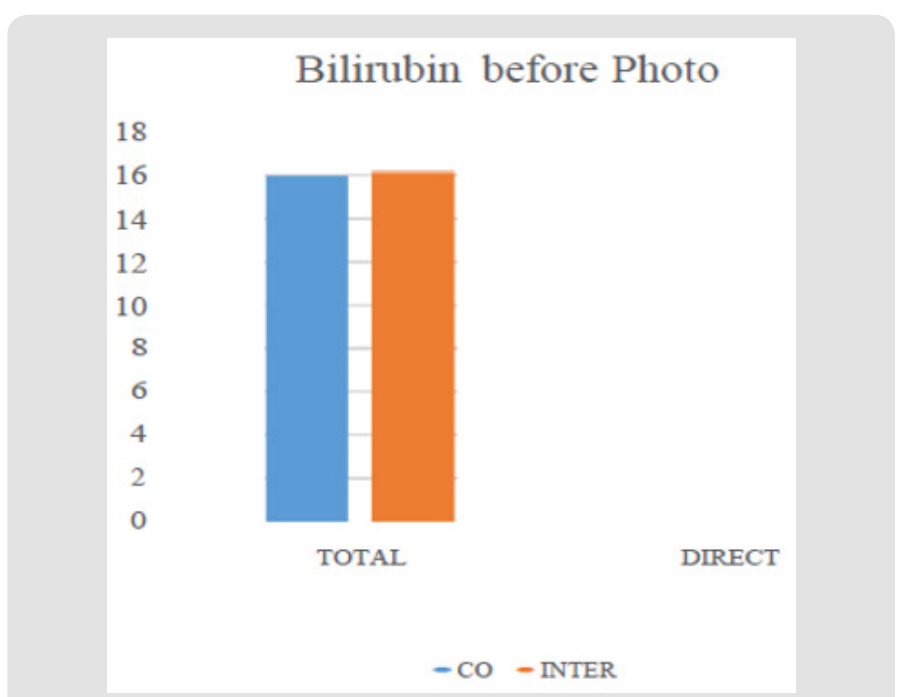

Figure 3: Bilirubin before phototherapy in two groups.

Table 2: Primary outcomes in two groups. Co: Continuous, Inter: Intermittent.

\begin{tabular}{|c|c|c|c|c|c|}
\hline \multicolumn{2}{|l|}{ Groups } & $\mathbf{N}$ & Mean & Std. Deviation & Std. Error Mean \\
\hline \multirow{2}{*}{$\begin{array}{l}\text { Total Bilirubin after } 24 \mathrm{hr} \text { photo } \\
\qquad(\mathrm{mg} / \mathrm{dl})\end{array}$} & Co & 30 & 12.29 & 1.15082 & 0.21011 \\
\hline & Inter & 30 & 13.3633 & 1.17985 & 0.21541 \\
\hline \multirow{2}{*}{$\begin{array}{l}\text { Total. Bilirubin after } 36 \mathrm{hr} \text { photo (mg/ } \\
\text { dl) }\end{array}$} & Co & 30 & 10.6933 & 0.9688 & 0.17688 \\
\hline & Inter & 30 & 10.5233 & 0.65109 & 0.11887 \\
\hline \multirow{2}{*}{ Duration of Phototherapy (hr) } & Co & 30 & 45.2667 & 16.39582 & 2.99345 \\
\hline & Inter & 30 & 46 & 11.82633 & 2.15918 \\
\hline \multirow{2}{*}{ Length of hospital stay (Day) } & Co & 30 & 2.3333 & 0.60648 & 0.11073 \\
\hline & Inter & 30 & 2.4667 & 0.9371 & 0.17109 \\
\hline
\end{tabular}




\begin{tabular}{|l|c|c|c|c|}
\hline \multirow{2}{*}{ Melatonin $(\mathrm{pg} / \mathrm{ml})$} & Co & 30 & 7.8267 & 9.11544 \\
\cline { 2 - 5 } & Inter & 30 & 9.4357 & 7.35862 \\
\hline
\end{tabular}

\section{Results}

The current RCT was conducted on 60 icteric term neonates. The demographic parameters did not differ significantly between the intermittent (group 1) and continuous (group 2) phototherapy groups. Among the 60 neonates, 29 (48.3\%) were female and 31 $(51.7 \%)$ male. There was no significant gender-difference between the two groups using the Fisher exact test. The mean age of the neonates at admission was 4.05 days (ranged 2 to 7 ). The mean weight of neonates at admission was $3289 \mathrm{~g}$, ranged 2500 to 4100 . The mean weight of neonates was $3322.33 \pm 5277$ and $3256.33 \pm 3829 \mathrm{~g}$ in the continuous and intermittent groups, respectively. There was no significant difference in the weight of neonates between the two groups $(\mathrm{P}>0.582)$. There was no significant difference in the $\mathrm{ABO}$ and Rh incompatibility between the two groups $(\mathrm{P}>0.5$ and $\mathrm{P}>0.2$, respectively). The mean total serum bilirubin at the onset of phototherapy was $15.97 \pm 1.55$ and $16.14 \pm 1.57 \mathrm{mg} / \mathrm{dL}$ in the continuous and intermittent groups, respectively. There was no significant difference in the mean total serum bilirubin at the onset of phototherapy between the two groups (P 0.676).Total serum bilirubin of the neonates in the two groups was also measured 6, 12, 24 and 36 hours after the onset of phototherapy if needed (Figure 4).

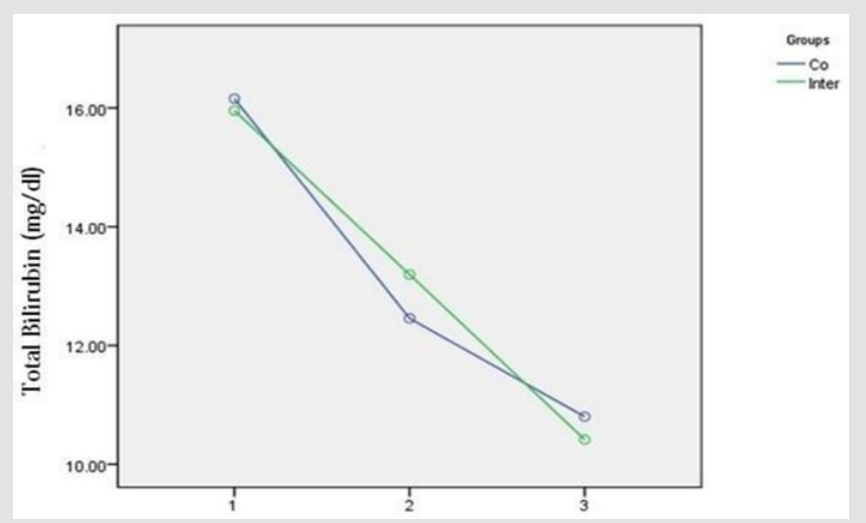

Figure 4: Comparison of total serum bilirubin $(\mathrm{mg} / \mathrm{dL})$ in two groups before phototherapy (1) and after 24 (2) and 36 hours (3) phototherapy.

The highest and lowest levels of bilirubin were 18 and 13mg/ $\mathrm{dL}$, respectively. In the current study, the mean total bilirubin after 24 hours was significantly lower in continuous phototherapy than in intermittent phototherapy groups $(\mathrm{P}<0.001)$. However, there were no significant differences between the two groups in the total serum bilirubin levels 36 hours after starting phototherapy $(\mathrm{P}=0.429)$. In other words, the reducing rate of total serum bilirubin was equal in both groups after 36 hours of phototherapy. The duration of hospital stay was $2.3 \pm 0.60$ and $2.46 \pm 0.93$ days in the continuous and intermittent groups, respectively $(\mathrm{P}=0.516)$. The duration of phototherapy was $45.26 \pm 16.39$ and $46 \pm 11.82$ hours in the continuous and intermittent groups, respectively $(\mathrm{P}=0.843)$. Although the melatonin level was higher in the intermittent group than in the continuous group, the differences were not statistically significant $(\mathrm{P}=0.455)$. No cases of death and kernicterus were observed in any of the groups.

\section{Discussion}

Although the current and previous studies had several common points, several differences were observed between them. In the current study, the continuous phototherapy group received phototherapy for 24 hours and the intermittent phototherapy group received phototherapy for 18 hours on and eight hours off (from 10:00 P.M to 6:00 A.M). Phototherapy lights were turned off for breastfeeding and changing for up to one hour in the two groups since it does not significantly affect the effect of phototherapy [1]. The phototherapy interval was applied to the intermittent group in order to save the circadian rhythm of infants; the melatonin levels of newborns were also measured to study the effect of phototherapy on circadian rhythm. Although the melatonin level was higher in the intermittent group than the continuous group, the difference was not statistically significant $(\mathrm{P}=0.455)$; the reason may be attributed to the short duration of phototherapy and the fact that if the duration of phototherapy was prolonged, the melatonin level might decrease. More clinical studies are needed to confirm these findings and a more extended study with more cases is suggested to prove this hypothesis. It is important, especially in prophylactic phototherapy for premature infants, to stay for a long time in NICU.

The total bilirubin level in the two groups after 24 hours of phototherapy was significantly different and continuous phototherapy reduced bilirubin more than intermittent phototherapy $(12.9 \pm 1.15$ and $13.36 \pm 1.17$ in the continuous and intermittent groups, respectively); however, the total bilirubin level in the two groups after 36 hours of phototherapy was not significantly different and continuous phototherapy reduced bilirubin as the much as intermittent phototherapy did $(10.69 \pm 0.96$ and $10.52 \pm 0.65$ in the continuous and intermittent groups, respectively). Results of the current study contribute to the ongoing research in this area. For instance, in the study by Monica Sachdeva et al. [12] intermittent phototherapy based on the 12:12 hours on-off schedule was compared with 24 hours continuous phototherapy and the results showed that the efficacy of intermittent phototherapy with 12:12 hours on-off cycle was as efficient as that of the continuous phototherapy ( $\mathrm{P}=0.002)$. In the current study, the duration of phototherapy cut off was too long, which had a potential of rebound hyperbilirubinemia. Lau and Fung concluded that there were no significant differences in serum bilirubin reduction between the two groups. 
They selected a schedule of one hour off and four hours on phototherapy for an intermittent group and achieved the same results of the continuous phototherapy group [13]. Niknafs et al. [14] found that intermittent phototherapy had the same results of continuous phototherapy. They selected a schedule of one hour on and one hour off for the intermittent group and a schedule of two hours on and half-an-hour off for the continuous group. In these two studies, the duration of phototherapy start/stop was too short, which might make the caregivers and nurses too tired; therefore, this schedule is not optimal. On the other hand, the circadian rhythm could not be preserved in these studies. These schedules were useful for neonates that cannot tolerate phototherapy well and may help to protect the mother-infant bounding, but maybe boring. In summary, with the current study protocol for intermittent phototherapy, besides an effective treatment for hyperbilirubinemia, the circadian rhythm can be preserved, the mother and infant bonding can be protected, and phototherapy unit's half-life can be saved. Maisels et al. [15] in their study concluded that phototherapy had complications such as oxidative stress and DNA damage in neonates. Therefore, intermittent phototherapy may decrease these complications by reduction of the duration of phototherapy.

\section{Conclusion}

According to the current study results, intermittent phototherapy was as effective as continuous phototherapy to decrease the total serum bilirubin level among icteric term infants. The melatonin level in the two groups did not show any significant differences. Intermittent phototherapy can be used as a routine procedure instead of continuous phototherapy in neonatal care units. More studies are needed to evaluate the phototherapy effects on the circadian rhythm of neonates.

\section{Declarations}

\section{Ethics approval and Consent to Participate}

The current study was approved by Ethics Committee of Shahid Beheshti University of Medical Sciences (ethical code: $66001089 / 27$ in 2016-11-13). The current study was registered in the Iranian Registry of Clinical Trials (IRCT2015112225184N1).

\section{Consent for Publication}

Not applicable.

\section{Availability of data and material}

Data sharing is not applicable to this article as no datasets were generated or analyzed during the current study.

\section{Authors' Contributions}

All stages of the design and commissioning of this study were done by the author.

\section{Acknowledgment}

Special thanks to Dr. Shahram Sabeti and Dr. Latif Gachkar from Loghman Hospital.

\section{Competing Interests}

The authors declared no conflict of interests.

\section{References}

1. Martin RJ, Fanaroff AA, Walsh MC (2015), Fanaroff and Martin's Neonatal-Perinatal Medicine: Diseases of the fetus and infant. Louis, (11 ${ }^{\text {th }}$ Edn.). MO: Elsevier Mosby (2): 1648-1649.

2. John P Cloherty, Eric C Eichenwald Manual of Neonatal Care. Neonatal, Hyperbilirubinemia, p: 32.

3. Lau and Fung (2001) AAP Subcommittee on Hyperbilirubinemia, Pediatrics 108(3): 763-765.

4. Fletcher MA, Mac Donald MG, Avery GB (2012) (Eds.), Avery's Neonatology Pathophysiology and Management of the Newborns. $\left(9^{\text {th }}\right.$ Edn.). Lippincott Williams and Wilkins, Philadelphia, PA, USA, pp. 311312 .

5. Hao H, Rivkees SA (1999) The biological clock of very premature primate infants is responsive to light. Proc Nat! Acad Sci USA 96(5): 2426-2429.

6. Hardeland Rudiger, Pandi Perumal SR, Cardinali Daniel P (2006) Melatonin. The International Journal of Biochemistry \& Cell Biology 38(3): 313-316.

7. Schomerus C, KorfHW (2005) Mechanisms regulating melatonin synthesis in the mammalian pineal organ. Annals of the New York Academy of Sciences 1057: 372-383.

8. Brainard GC, Hanifin JP, Greeson JM, Byrne B, Glickman G, et al. (2001) Action spectrum for melatonin regulation in humans: evidence for a novel circadian photoreceptor. J.Neurosci 21(16): 6405-6412.

9. Hickman AB, Namboodiri MA, Klein DC, Dyda F (1999) The structural basis of ordered substrate binding by serotonin $\mathrm{N}$-acetyltransferase: enzyme complex at $1.8 \mathrm{~A}$ resolution with a bisubstrate analog. Cell 97(3): 361-369.

10. Carrillo-Vico A, Guerrero JM, Lardone PJ, Reiter RJ (2005) A review of the multiple actions of melatonin on the immune system. Endocrine 27(2): 189-200.

11. Macchi MM, Bruce JN (2004) Human pineal physiology and functional significance of melatonin. Front Neuroendocrinol 25(3-4): 177-195.

12. Sachdeva M, Murki S, Oleti TP, Kandraiu H (2015) Intermittent versus continuous phototherapy for the treatment of neonatal hyperbilirubinemia A RCT, Eur Pediatr 174(2): 177-181.

13. Lau SP, Fung KP (1984) Serum bilirubin kinetics in intermittent phototherapy of physiological jaundice. Arch Dis Child 59(9): 892-894.

14. Pedram Nik, Abdol Ali Mo, Mohammad Hossein To, Bahare Ba, Nikoo $\mathrm{Ni}$ (2008) Intermittent versus Continuous Phototherapy for Reducing Neonatal Hyperbilirubinemia. Iran J Pediatr 18(3): 251-256.

15. Maisels MJ, Newman TB (2012) Phototherapy and other treatments. In Stevenson DK, Maisels MJ, Watchko JF, (Eds.). Care of the jaundiced neonate. McGraw Hill, New York, USA, pp. 195-227. 
ISSN: 2574-1241

DOI: 10.26717/BJSTR.2020.24.004017

Roya Taheritafti. Biomed J Sci \& Tech Res

(c) (P) This work is licensed under Creative BY Commons Attribution 4.0 License

Submission Link: https://biomedres.us/submit-manuscript.php

$\begin{array}{ll}\text { BIOMEDICAL } & \text { Assets of Publishing with us } \\ \text { RESEARCHES } & \text { - Global archiving of articles } \\ & \text { - Immediate, unrestricted online access } \\ & \text { - Rigorous Peer Review Process } \\ & \text { - Anttps://biomedres.us/ }\end{array}$

